\title{
miR-124 promotes apoptosis and inhibits the proliferation of vessel endothelial cells through P38/MAPK and PI3K/AKT pathways, making it a potential mechanism of vessel endothelial injury in acute myocardial infarction
}

\author{
WEIMIN MA ${ }^{1}$, XIN ZHANG ${ }^{2}$ and YANG LIU ${ }^{1}$ \\ Departments of ${ }^{1}$ Critical Care Medicine and ${ }^{2}$ Pediatrics, Weihai Central Hospital, Weihai, Shandong 264200, P.R. China
}

Received December 22, 2020; Accepted August 11, 2021

DOI:10.3892/etm.2021.10819

\begin{abstract}
Due to its rapid onset and high rates of fatality, acute myocardial infarction (AMI) has long been one of the most fatal diseases among all types of heart diseases. Therefore, intensive research efforts have been focused on understanding AMI's potential pathogenesis to seek effective treatment options. In the present study, 20 peripheral blood samples were collected from patients with AMI, after which reverse transcription-quantitative PCR analysis revealed that microRNA (miR)-124 levels in the peripheral blood of patients with AMI was significantly elevated compared with that in the control group. In vitro, a model using pcDNA3.1-miR-124 transfected human umbilical vein endothelial cells (HUVECs) indicated that overexpression of miR-124 could significantly promote the apoptosis and suppress the proliferation of HUVECs using flow cytometry, TUNEL assay and Cell Counting Kit- 8 assays. Based on the present findings, RNA samples of HUVECs overexpressing miR-124 was extracted and sequenced to explore the gene expression profile after miR-124 overexpression. Gene Set Enrichment Analysis (GSEA) analysis revealed that the downregulated genes were mainly enriched in signaling pathways, such as PI3K-AKT, whilst the upregulated genes were mainly enriched in metabolism-related signaling pathways, such as the metabolism of xenobiotics by cytochrome $\mathrm{P} 450$ pathway. Additionally, Rideogram software was used to determine the chromosomal localization of the differentially expressed genes. The results demonstrated that they were distributed on all chromosomes except for chromosome Y. In addition, characteristic profiles of the differentially expressed genes caused by miR-124 overexpression were analyzed using Connectivity
\end{abstract}

Correspondence to: Dr Yang Liu, Department of Critical Care Medicine, Weihai Central Hospital, 3 West Shandong Road, Weihai, Shandong 264200, P.R. China

E-mail: liuyang_wenzhang@163.com

Key words: microRNA-124, acute myocardial infarction, RNA sequencing, PI3K/AKT signal pathway
Map. In total, two medicines, anisomycin and sanguinarine, which function as p38/MAPK signaling agonists that can inhibit angiogenesis, presented with the highest enrichment scores. Together with the GSEA results, which indicated that the differentially expressed genes were mainly enriched in the angiogenesis-inhibiting PI3K/AKT signaling pathway, the present study reported that high expression of miR-124 was negatively associated with patients with AMI, promoting the apoptosis and suppressing the proliferation of vessel endothelial cells.

\section{Introduction}

Among the large number of chronic diseases worldwide, myocardial infarction is one of the most lethal. According to statistical data, there were $\geq 2$ million cases of myocardial infarction in China in 2015, with 500,000 new cases every year and a falling trend in the age of patients (1). Therefore, the pathophysiology of this disease has long been the subject of intense scientific research. According to their pathogenic profiles, acute myocardial infarction (AMI) can be roughly divided into the following types: i) Type 1 myocardial infarction, which is caused by the interruption of or insufficient blood supply to the myocardium, mainly due to acute coronary artery occlusion associated with atherosclerosis or superimposed thrombosis (2), ii) type 2 myocardial infarction, which is normally caused by the metabolic capacity of cardiomyocytes far exceeding blood supply, where inflammation and fever increase the metabolic demand of tissues and organs, thereby increasing the heart rate and ultimately compromising coronary perfusion (3); and iii) myocardial infarctions caused by cytokine storms, which can inhibit aerobic respiration in the mitochondria and subsequent acute heart failure (4). In clinical practice, the main cause of AMI remains to be acute viral or bacterial infections caused by influenza, pneumonia and acute bronchitis (5). However, the precise molecular mechanism underlying AMI remain unclear.

An increasing number of studies have reported that miRNAs can serve important roles in the occurrence of diseases $(6,7)$. miRNAs belong to a family of post-transcriptional regulators for gene expression. These small non-coding RNAs, which are 20-25 nucleotides in length, bind to target recognition sites 
(seed sequence) in the 3'-untranslated regions of their mRNA transcript, leading to mRNA degradation and/or inhibition of protein translation (8).

A number of studies have demonstrated that the cardiovascular system exhibits high expression of miRNAs, which are related to the occurrence and development of heart failure, atherosclerosis, arrhythmia and coronary heart disease $(9,10)$. Additionally, a growing number of miRNAs have been reported to regulate angiogenesis after AMI. Angiogenesis, which is the formation of new capillaries from pre-existing vessels, that occurs in the ischemic area after myocardial infarction (MI) promotes cardiomyocyte survival and is considered to be essential to the recovery of patients with MI (11). miR-1 was discovered to be a potential biomarker for AMI diagnosis and prognosis, since it was released within $3 \mathrm{~h}$ after the onset of acute chest pain (12). It has also been demonstrated that miR-199 and miR-208 exerted similar roles as miR-1 in assisting the clinical diagnosis of AMI (13). In addition, it has been reported that increased levels of serum miR-214 is closely related to the occurrence of cardiomyocyte apoptosis in elderly patients with AMI (14). Mechanistically, other studies have demonstrated that manipulating the expression of several miRNAs can exert direct effects on angiogenesis. Yucel and Sahin (15) reported that silencing of miR-10b expression could significantly inhibit the angiogenesis of vessel endothelial cells. In colon cancer, miR-524-5p has been found to target WNK lysine deficient protein kinase 1, thereby inhibiting the expression of cell epidermal growth factor to inhibit cell angiogenesis (16). In addition, another study revealed that silencing of miR-212/132 expression could significantly reduce the ability of human vessel endothelial cells to form blood vessels (17).

In the present study, clinical blood samples of patients with AMI were analyzed to measure the expression of miR-124. An in vitro cell model using pcDNA3.1-miR-124 transfected human umbilical vein endothelial cells (HUVECs) was constructed in the present study. The apoptosis and proliferation of HUVECs was assessed using flow cytometry, TUNEL and Cell Counting Kit-8 (CCK-8) assays. Subsequently, the transcriptome of HUVECs overexpressing miR-124 was sequenced and analyzed. Exploring the effect of miR-124 overexpression on gene expression in vascular endothelial cells may provide new avenues for the development of novel treatment strategies for AMI.

\section{Materials and methods}

Patient information. Blood samples were collected from 20 patients (male, 16; female, 4; average age, 60.2 \pm 3.7 years) admitted to the Weihai Central Hospital with myocardial infarction between March 2016 and June 2017. In addition, blood samples were collected at the Weihai Central Hospital from 14 healthy volunteers between March 2016 and June 2017 (male, 11; female, 3; aged >55 years; average age, $60.5 \pm 3.1$ years; no symptoms or history of AMI). Blood samples were collected from healthy people on admission and from patients with myocardial infarction 7 days after the symptoms of myocardial infarction had been controlled. The study protocol was approved by the Ethics Committee of Weihai Central Hospital (no. WKY-2019-012). Informed consent was provided by all patients, and written permission was obtained before inclusion. Patients were included in the current study if they exhibited ST segment elevation myocardial infarction or non-ST segment elevation myocardial infarction. Healthy individuals were included in the present study if they were $>55$ years of age. Patients with AMI were excluded if they exhibited: Diabetes mellitus, infectious disease and autoimmune disease. Healthy patients were excluded if they exhibited diabetes mellitus, myocardial infarction, and infectious and autoimmune diseases.

Extraction of free RNA from peripheral blood and reverse transcription-quantitative PCR (RT-qPCR) identification. Blood was collected in a 5-ml heparin anticoagulation tube and immediately used to extract free RNA (GSPureTM Blood RNA Isolation kit, Geneseed; cat. no. E0203). When extracting miRNA, the ethanol concentration in the cleaning buffer was increased to $80 \%$ to increase the final miRNA concentration. The RNA was reverse transcribed using a reverse transcription kit at $37^{\circ} \mathrm{C}$ for $60 \mathrm{~min}$, followed by termination at $85^{\circ} \mathrm{C}$ for $5 \mathrm{~min}$ to inactivate the enzymes (Takara Bio, Inc.; cat. no. 638315). In total, $25 \%$ of the RT product was mixed with a pre-formulated 2X SYBR Green PCR mix containing the miR-124 RT-qPCR primers, which were dissolved to $20 \mu \mathrm{l}$ with $\mathrm{ddH}_{2} \mathrm{O}$ (Roche Diagnostics; cat. no. 06402712001). Amplification was performed to collect the dissolution curve with the following thermocycling conditions: $94^{\circ} \mathrm{C}$ for $5 \mathrm{~min}$; 40 cycles of $94^{\circ} \mathrm{C}$ for $2 \mathrm{~min}, 60^{\circ} \mathrm{C}$ for $1 \mathrm{~min}$ and $72^{\circ} \mathrm{C}$ for $2 \mathrm{~min}$; last step at $72^{\circ} \mathrm{C}$ for 2 min. U6 mRNA was used as an internal reference and the relative expression of miR-124 was calculated using the $2^{-\triangle \Delta C q}$ method (18). The primers used for PCR were as follows: p38 forward, 5'-AGGGCGATGTGACGTTT-3' and reverse, 5'-CTGGCAGGGTGAAGTTGG-3'; AKT forward, 5'-TGG ACTACCTGCACTCGGAGAA-3' and reverse, 5'-GTGCCG CAAAAGGTCTTCATGG-3'; PI3K forward, 5'-GAAGCA CCTGAATAGGCAAGTCG-3' and reverse, 5'-GAGCATCCA TGAAATCTGGTCGC-3'; $\beta$-actin forward, 5'-AAAGACCTG TACGCCAACAC-3' and reverse, 5'-GTCATACTCCTGCTT GCTGAT-3'; miR-124 forward, 5'-TAAGGCACGCGGTGA ATGCC-3' and reverse, 5'-CAGGTCCAGTTTTTTTTTTTTT TTVN-3'; U6 forward, 5'-GCTTCGGCAGCACATATACTA AAAT-3' and reverse, 5'-CGCTTCACGAATTTGCGTGTC AT-3'.

Cell culture, miR-124 overexpression and RT-qPCR. HUVECs were obtained from the American Type Culture Collection. The cells were cultured in DMEM medium (Hyclone, Cytiva) containing 10\% (v/v) FBS (Gibco; Thermo Fisher Scientific, Inc.) and $1 \%$ penicillin, which were incubated at $37^{\circ} \mathrm{C}$ in an atmosphere with $10 \% \mathrm{CO}_{2}$.

To construct the pcDNA3.1-miR-124 vector, cDNA encoding pri-miR-124 (accession no. MI0000443) was artificially synthesized and cloned into the pcDNA3.1 vector (Sangon Biotech Co., Ltd.). Samples were then transfected with $0.5 \mu \mathrm{g}(1 \mu \mathrm{g} / \mu \mathrm{l})$ pcDNA3.1-miR-124 or pcDNA3.1 vector into HUVECs using Lipofectamine ${ }^{\circledR} 2000$ (Invitrogen; Thermo Fisher Scientific, Inc.) according to the manufacturer's protocol at $37^{\circ} \mathrm{C}$ in the presence of $5 \% \mathrm{CO}_{2}$ for $24 \mathrm{~h}$. The empty vector pcDNA3.1 was similarly transfected into HUVECs as negative control (NC group). RNA was extracted from HUVECs by cell lysis with the RNeasy Mini kit (Qiagen 
$\mathrm{GmbH}$; cat. no. 74104) lysis buffer on ice, after washing with ice-cold PBS. RNA was extracted with a spin column Qiagen $\mathrm{GmbH}$ ). Transcriptor First Strand cDNA Synthesis Kit (Roche Diagnostics) was used to reverse transcribed the isolated mRNA into complementary DNA at $65^{\circ} \mathrm{C}$ for $10 \mathrm{~min}$.

The One Step miR cDNA Synthesis kit (cat. no. D1801; Xinhai Gene Testing Co., Ltd.) was used for the reverse transcription of miR-124 at $37^{\circ} \mathrm{C}$ for $60 \mathrm{~min}$.

The expression level of miR-124 was measured by qPCR performed with SYBR Green Kit (Takara Bio, Inc.). U6 was used as an internal reference, and the relative expression of miR-124 was calculated using the aforementioned $2^{-\Delta \Delta \mathrm{Cq}}$ method.

HUVEC proliferation and apoptosis assay. The CCK-8 assay (Bimake; cat. no. B34302) was used to evaluate the changes in HUVEC proliferation after miR-124 overexpression. Briefly, after transfection with pcDNA3.1-miR-124 or pcDNA3.1, HUVECs were seeded into a 96-well plate at a density of 5,000 cells/well, before cell viability was analyzed. A total of $20 \mu \mathrm{l}$ CCK-8 reagent was added into each well and incubated at $37^{\circ} \mathrm{C}$ for $4 \mathrm{~h}$. The optical density at $450 \mathrm{~nm}$ was read with a microplate reader (Thermo Fisher Scientific, Inc.). In total, three independent experiments were performed.

For the TUNEL assay, HUVECs were fixed with $4 \%$ paraformaldehyde for $20 \mathrm{~min}$ at room temperature. Cells were washed three times with PBS and stained with $50 \mu 1$ TUNEL reaction mixture containing FITC-labelled dUTP and TdT for $1 \mathrm{~h}$ at $37^{\circ} \mathrm{C}$ (In situ Cell Death Detection kit, Roche Diagnostics; cat. no. 11684795910). Each sample was randomly counted in five fields. The TUNEL index $(\%)$ is the average ratio of the number of TUNEL-positive cells divided by the total number of cells under bright-field.

Flow cytometry was used to measure the cell apoptosis status following miR-124 overexpression in HUVECs. The apoptosis rate was evaluated using Annexin V-FITC/PI Apoptosis Detection kit (Tianjin Sungene Biotech Co., Ltd; cat. no. AO2001-02P-G) according to the manufacturer's protocol. Briefly, after transfection with pcDNA3.1-miR-124 or pcDNA3.1, the cells were collected, washed with PBS and resuspended in $500 \mu \mathrm{l}$ binding buffer. Subsequently, $100 \mu \mathrm{l}$ $1 \times 10^{6} / \mathrm{ml}$ cells were incubated at room temperature for $1 \mathrm{~h}$ with $5 \mu \mathrm{l}$ Annexin V-FITC and $5 \mu \mathrm{l}$ PI in the dark, before analysis by flow cytometry (BD FACSCanto ${ }^{\mathrm{TM}}$; BD Biosciences). Data were analyzed using FlowJo software (FlowJo LLC; version no. V10).

Western blot assay. Total proteins from HUVECs were extracted by lysis with ice-cold RIPA buffer (Pierce; Thermo Fisher Scientific, Inc.; cat. no. 89900) with protease inhibitors (Roche Diagnostics; cat. no. 04693159001). The protein concentration of the sample was determined using a BCA assay (Thermo Fisher Scientific, Inc.; cat. no. 23225). Electrophoresis was conducted with equal amount of protein samples $(40 \mu \mathrm{g})$ by $10 \%$ SDS-PAGE, before being transferred onto PVDF membranes. The membranes were incubated in $5 \%$ fat-free milk in PBST (0.1\% Tween-20) at room temperature for $30 \mathrm{~min}$. Then incubated with primary antibodies against PI3K (1:1,000; Cell Signaling Technology, Inc.; cat. no. 4249), AKT (1:1,000; Cell Signaling Technology, Inc.; cat. no. 9272), p38 (1:1,000; Cell Signaling Technology, Inc.; cat. no. 8690) and $\beta$-actin as a loading control (1:1,000; Sangon Biotech Co., Ltd.; cat. no. D191047) at $4^{\circ} \mathrm{C}$ overnight. After washing 3 times with PBS-Tween (0.1\% Tween-20), membranes were incubated with HRP-conjugated goat anti-rabbit IgG secondary antibodies (1:10,000; Cell Signaling Technology, Inc.; cat. no. 7074S) at room temperature for $2 \mathrm{~h}$. Protein bands were visualized using the chemiluminescence kit (Millipore Sigma; cat. no. WBKLS0500).

Sequencing and bioinformatics analysis. Total RNA was isolated using RNeasy Mini Kit(Qiagen) according to the manufacturer's protocol. RNA with an RNA integrity number $>8$, as assessed using the Agilent Technologies 2100 Bioanalyzer (Agilent Technologies, Inc.), was used to prepare the c-DNA library using the Hieff NGS ${ }^{\circledR}$ MaxUP II DNA Library Prep kit for Illumina (Shanghai Yeasen Biotechnology Co., Ltd.; cat. no. 12300) following the manufacturer's protocols. Sequencing was performed by Novogene Co., Ltd. using the HiSeq X Ten system (v 2.5; Illumina, Inc.) for 2x150 cycles (paired-end read length of $150 \mathrm{bp}$ ). The loading DNA concentration was $300 \mathrm{pM}$. Concentration of libraries were determined using the Agilent Technologies 2100 Bioanalyzer (Agilent Technologies, Inc.). Reads that were contaminated by adaptors, exhibited a Phred quality score (19) $<5$ accounting for $>50 \%$ of total bases and reads with an $\mathrm{N}$ content $>10 \%$ were removed using Fastp software (https://github.com/OpenGene/ fastp; version 0.20.0). Clean reads were mapped to the human genome (hg19) using TopHat software (v2.1.0) (20). Kallisto (version v0.43.0; https://pachterlab.github.io/kallisto) (21) was used to quantify the reads obtained by sequencing and the sleuth package (22) in the R language was used for differential expression analysis (22). PC analysis was carried out using the plotPCA (23), whereas the heatmap was drawn according to gene expression using the pheatmap package (https://CRAN.R-project.org/package=pheatmap) in the R language. The ggplot2 package (https://CRAN.R-project.org/ package $=$ ggplot2) was used to draw the volcano plots. Pearson's correlations were calculated using the R (v3.6) core function. The threshold for differentially expressed genes was $\mathrm{q}$ val $<0.1$ and $\operatorname{abs}\left(\log _{2} \mathrm{FC}\right)>0$. The RIdeogram package was used to determine the chromosomal localization of the differentially expressed genes (24). The CMAP database collects the gene expression profiles of human cells treated with multiple single FDA-approved small-molecule drugs. The gseKEGG function in the clusterprofiler package (25) was used to perform Gene Set Enrichment Analysis (GSEA) of the Kyoto Encyclopedia of Genes and Genomes pathway (threshold, q-values <0.05) and Connectivity Map (CMAP) analysis on the expression matrix (threshold, $\mathrm{P}<0.05$ ).

Statisticalanalysis. Graphpad Prism Software v.8.0(GraphPad Software, Inc.) was used for statistical analysis. All experiments were performed in triplicates and data is presented as the mean \pm SD. Single comparisons were performed using paired or unpaired Student's t-test. The statistical significance data involving $\geq$ three groups and $\geq$ two treatments was assessed by two-way ANOVA with Bonferroni post hoc test. $\mathrm{P}<0.05$ was considered to indicate a statistically significant difference. 


\section{Results}

Free miR-124 levels in the blood of patients with AMI is significantly higher compared with those in healthy individuals. The peripheral blood of patients with AMI and of healthy controls was collected. When extracting miRNA, the ethanol concentration in the cleaning buffer was increased to $80 \%$ to increase the final miRNA concentration. As presented in Fig. 1, the expression level of miR-124 in the samples of the patients with AMI was significantly higher compared with that in the healthy control group.

Overexpression of miR-124 can promote the apoptosis of HUVECs and suppress their proliferation ability. Subsequently, the effects of miR-124 on the proliferation and apoptosis in HUVECs were evaluated. First, RT-qPCR was performed on samples from HUVECs that were transfected with miR-124 overexpression plasmid or empty pcDNA3.1 plasmid. The experimental results indicated that the expression of miR-124 in the group transfected with the overexpression plasmid was significantly higher compared with that in the NC group (Fig. 2A), suggesting that miR-124 was successfully overexpressed in HUVECs. The proliferation ability of miR-124-overexpressing HUVECs was also significantly lower compared with that in the NC group after 5 days (Fig. 2B). TUNEL assay was subsequently used to detect the effect of miR-124 overexpression on the apoptotic ability of HUVECs. In terms of apoptosis, the number of TUNEL-positive cells in the miR-124 overexpression group was significantly higher compared with that in the empty pcDNA3.1 group (Fig. 2C), suggesting that overexpression of miR-124 may promote apoptosis in HUVECs. In addition, flow cytometry was also performed to measure the apoptosis, where miR-124 overexpression significantly promoted cell apoptosis compared with that in the NC group (Fig. 2D).

Overexpression of $\mathrm{miR}-124$ can significantly change the gene expression profile of HUVECs. To investigate the expression characteristics of genes related to miR-124, RNA sequencing was performed on the NC and the miR-124 overexpression groups. Principal component analysis was then performed to determine the effect of miR-124 overexpression in HUVEC cells. The results indicated that the two methods of transfections, miR-124 overexpression and NC, formed a distinct cluster, in which the expression patterns of the two groups were different (Fig. 3A). The heatmap was generated using Pearson's correlation analysis, where differential gene expression was calculated between samples. The results demonstrated that similarity within miR-124-overexpressing groups is higher than the similarity between miR-124-overexpressing group and empty pcDNA3.1 group which means that they have distinct expression patterns (Fig. 3B). The present results indicated that the overexpression of miR-124 can significantly alter the gene expression profile of HUVEC.

Screening of differentially expressed genes and their chromosomal location analysis. When using the Sleuth package to calculate differentially expressed genes, $\mathrm{q}$ val $<0.1$ and $\mathrm{abs}\left(\log _{2} \mathrm{FC}\right)>0$ were used as the screening conditions. The results revealed a total of 238 differential genes, of which

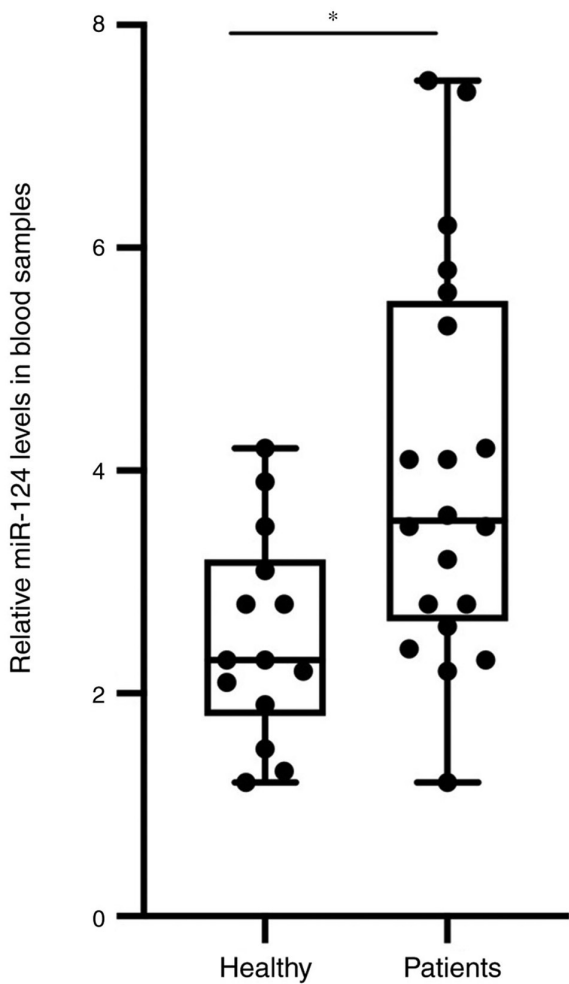

Figure 1. Expression level of miR-124 in blood samples of patients and healthy individuals as detected by reverse transcription-quantitative PCR. miR-124 levels were significantly higher in patients with acute myocardial infarction compared with those in the healthy individuals. ${ }^{*} \mathrm{P}<0.01$ (unpaired t-test). miR, microRNA.

103 genes were downregulated and 135 genes were upregulated in the miR-124-overexpressing cells (Fig. 4A). In addition, cluster analysis was performed on these differentially expressed genes. As shown in Fig. 4B, the differentially expressed genes presented significantly different expression patterns in the miR-124-overexpressing and NC groups. For example, the most downregulated genes were SLC48A1, ALPP and PPL. The most upregulated genes were IL1A, HMGA2 and SPOCK1. Chromosomal location analysis using the RIdeogram package was used to determine the chromosomal localization of the differentially expressed genes (Fig. 4C). The differentially expressed genes were found to be scattered on all chromosomes except the Y chromosome. Among them, chromosome 1 contained the highest distribution of genes with 29 (accounting for 12.2\%), followed by $22 \%$ on chromosome 12 and $16 \%$ on chromosome 19 . There were no differentially expressed genes on chromosome $\mathrm{Y}$, which could mean that miR-124 overexpression did not mediate any effects on gene expression on the Y chromosome.

Enrichment analysis and CMAP analysis of differentially expressed genes. Small-molecule drugs causing gene expression changes in human cells similar to miR-124 overexpression were researched using the CMAP database, where total of 90 small-molecule drugs with statistically significant similarity were found (Fig. 5A). This analysis was performed to screen for drug candidates that could block miR-124 from causing vascular endothelial cell damage. Among the three drugs with enrichment scores $>0.9$, anisomycin and sanguinarine are reported to be agonists of the p38/MAPK signaling 
A

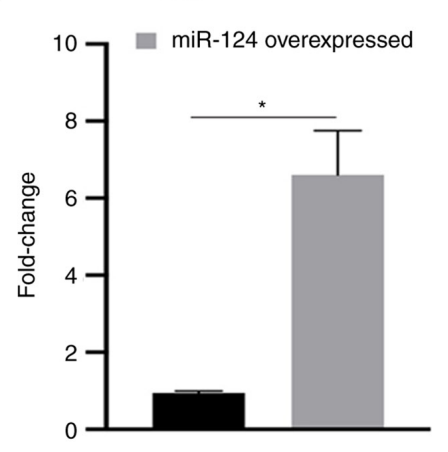

B

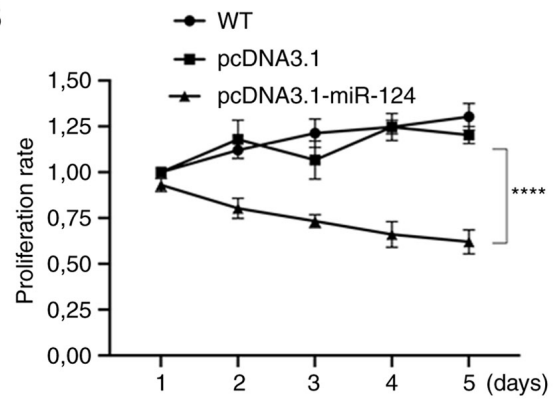

C

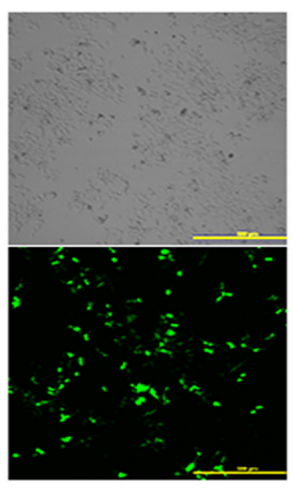

miR-NC

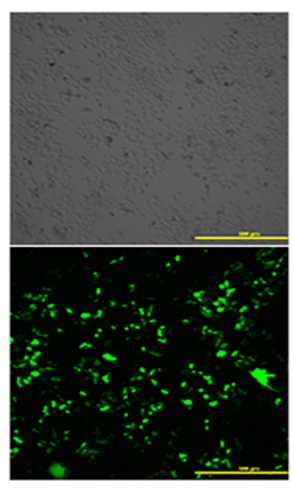

miR-124 overexpressed

D

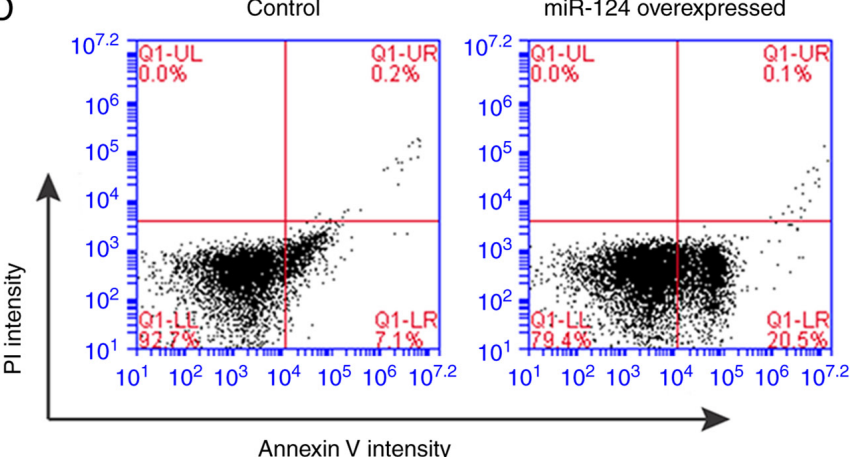

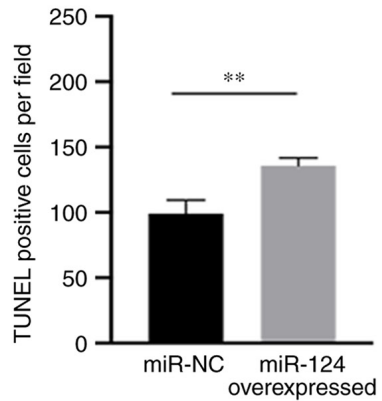

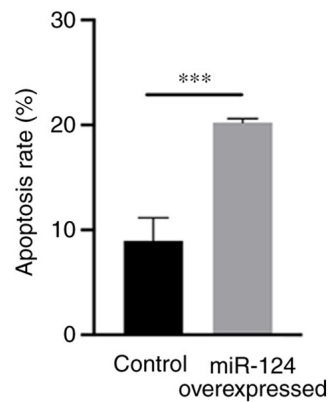

Figure 2. Effect of miR-124 overexpression on human umbilical vein endothelial cell proliferation and apoptosis. (A) Expression of miR-124 in human umbilical vein endothelial cells overexpressing miR-124 or an NC (pcDNA3.1 vector) as detected by reverse transcription-quantitative PCR "P<0.05 (paired t-test). (B) Effect of miR-124 overexpression on cell proliferation. ${ }^{* * * * * *} \mathrm{P}<0.0001$ (paired t-test) vs. NC(pcDNA3.1 vector) on day 5. (C) Effect of miR-124 overexpression on cell apoptosis by TUNEL assay. ${ }^{* * *} \mathrm{P}<0.05$ (paired t-test). (D) Effect of miR-124 overexpression on cell apoptosis by flow cytometry analysis. ${ }^{* * * *} \mathrm{P}<0.05$ (paired t-test). NC, negative control; miR, microRNA.

pathway and could inhibit angiogenesis, an unfavorable factor for the recurrence of myocardial infarction (26). This suggested that the results of CMAP were relatively accurate. The present analysis also suggested that the three drugs with the lowest enrichment score, CP-645525-01, rifabutin and quinostatin, had the potential to promote angiogenesis and to reduce the risk of myocardial infarction recurrence (Fig. 5A). GSEA was performed to further investigate the inhibitory effect of miR-124 on angiogenesis. As presented in Fig. 5B, 17 pathways (qvalue $<0.05$ ) were obtained, which were associated with the decrease of HUVEC proliferation after miR-124 overexpression. Among them, the pathway that included the most related genes was the PI3K-AKT signaling pathway. The peak in the GSEA results of the PI3K-AKT signaling pathway shifted left, with NES $=1.78$, suggesting that most of the genes in the PI3K-AKT signaling pathway were downregulated. Meanwhile, the upregulated genes were mainly enriched in various metabolism-related signaling pathways, including the metabolism of xenobiotics via the cytochrome P450 pathway.

miR-124 can influence the p38/MAPK and PI3K/AKT pathways. RT-qPCR and western blotting assays were performed to explore the expression change of key components of the p38/MAPK and PI3K/AKT pathways caused by miR-124 overexpression in HUVECs. As presented in Fig. 6, PI3K, AKT and p38 expression levels significantly decreased on both mRNA and protein levels compared with that in the NC group. The present results suggest that miR-124 can influence the p38/MAPK and PI3K/AKT pathways. 
A

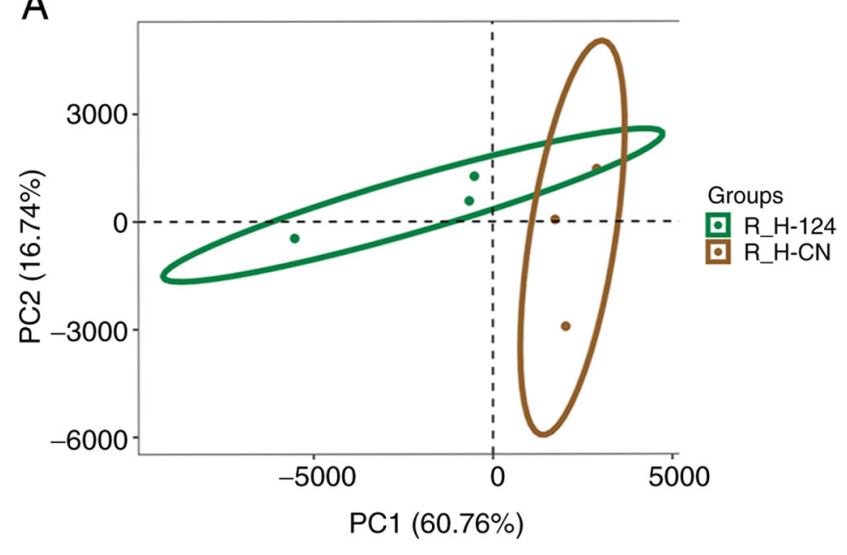

B

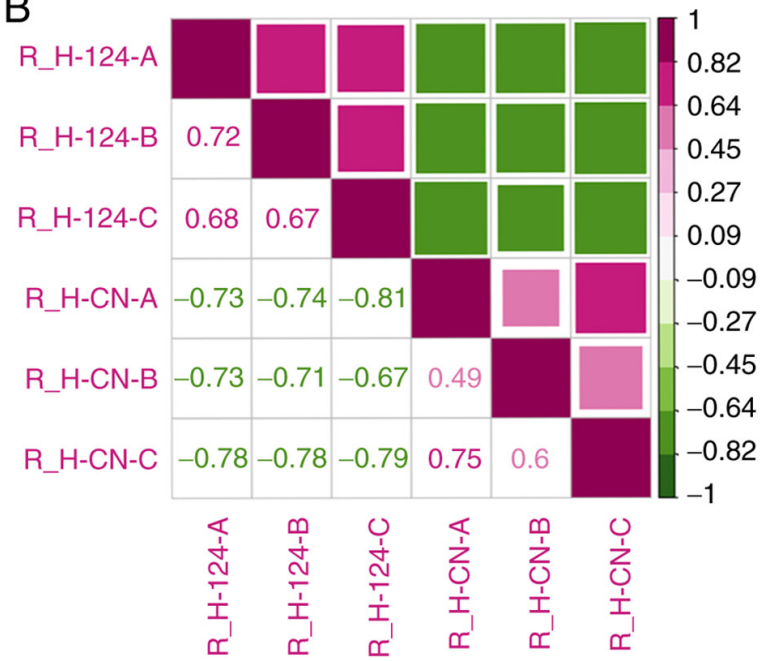

Figure 3. Analysis of gene expression patterns. (A) plotPCA was used to analyze human umbilical vein endothelial cell RNA-seq samples that demonstrated tight grouping of biological replicates. The green oval represents the $95 \%$ confidence interval of principal component distribution in the microRNA 124 -overexpression samples. The brown oval represents the 95\% confidence interval of principal component distribution in NC samples. (B) Pearson's correlation analysis was performed using the R (v3.6)'s core function. PCA, principal component analysis; NC, negative control; miR, microRNA; R_H-124-A, R_H-124-B, R_H-124-C represent microRNA 124-overexpression samples; R_H-CN-A, R_H-CN-B, R_H-CN-C represent negative control samples. R represents the use of R language for data analysis, $\mathrm{H}$ represents $\mathrm{HUVEC}$ cells, and A, B and C represent three different repeated samples. $\mathrm{CN}$, negative control.

\section{Discussion}

In the present study, the effects of miR-124 overexpression on gene expression in HUVECs were explored. Previous studies on the role of miR-124 suggest that it can regulate cardiomyocyte apoptosis and myocardial infarction (27). Furthermore, it was shown that peripheral blood miR-124 can be used to predict AMI (28). In AMI, the regenerative capacity of blood vessels is an important indicator for evaluating myocardial recovery $(29,30)$. Previous studies found miR-124 to be mainly expressed in the human brain, suggesting that its functions are considered to be related to neural differentiation $(31,32)$. A previous study demonstrated that miR-124 could target the nuclear receptor subfamily 3 group $\mathrm{C}$ member 2 gene and regulate the renin-angiotensin-aldosterone system, which is mainly associated with the regulation of blood pressure (33). In addition, it was demonstrated that miR-124 expression is associated with impaired cardiac function (27). For this reason, clinical samples were obtained to detect the expression of miR-124 in patients with AMI in the present study, which revealed that miR-124 expression in patients with AMI was significantly higher compared with that in the healthy control group. Therefore, the present results strongly indicated that miR-124 function is related to AMI pathophysiology.

The present study also found that miR-124 overexpression markedly altered gene expression in HUVECs. Compared with those in the $\mathrm{NC}$ group, there were $\geq 238$ differentially expressed genes; which were relatively enriched in the PI3K/AKT signaling pathway. AKT serves an important role in regulating postnatal cardiac development, myocardial angiogenesis and cell death in cardiac myocytes (34). In a clinical setting, PI3K/AKT signaling activators are frequently used as therapeutic options for clinical improvement of cardiac related diseases (35). By contrast, an increasing number of biological macromolecules, such as 17 beta-estradiol (36) and
TO901317 (37), have been found to activate the PI3K/AKT signaling pathway. It was previously reported that high mobility group box protein 1 could protect the heart from ischemia-reperfusion injury by the PI3K/AKT pathway-mediated upregulation of VEGF expression (38). There are a number of other similar reports in which there is a close correlation between the expression of PI3K/AKT and the heart-related disease (39-42).

The restoration of myocardial blood supply is an important therapeutic strategy for AMI recovery (39). Therefore, the extent of angiogenesis mediated by vascular endothelial cells has become an important indicator for evaluating the prognosis of AMI (43). The PI3K/AKT signaling pathway is an important anti-apoptotic signaling pathway, for example, in colorectal, gastric and breast cancer as well as others (44). Blocking the PI3K/AKT/mTOR signaling pathway can not only induce cell cycle arrest, apoptosis and autophagy, but can also inhibit prostate tumor cell angiogenesis (45). In this regard, a number of organic and biological agents have been reported to regulate angiogenesis through the expression of genes in this signaling pathway. Celastrol can inhibit the angiogenic ability of glioma by inhibiting the expression and activation of hypoxia inducible factor- $1 \alpha$, PI3K, AKT and mTOR, thereby inhibiting angiogenesis (46). Additionally, miR-9 can regulate the PI3K/AKT signaling pathway by targeting the transient receptor potential cation channel subfamily $\mathrm{M}$ member 7 gene to promote the angiogenesis of endothelial progenitor cells (47). miR-221 can also regulate the function of endothelial cells, including HUVECs, through the PI3K/AKT signaling pathway (48). Overexpression of miR-221 can significantly improve the angiogenic ability of HUVECs, whilst the inhibition of miR-221 directly increased the apoptosis rate of HUVECs (48). Low-level laser therapy has also been shown to increase the phosphorylation levels of PI3K, AKT and mTOR proteins, where weakening or blocking the PI3K signaling pathway could significantly reduce the 
A

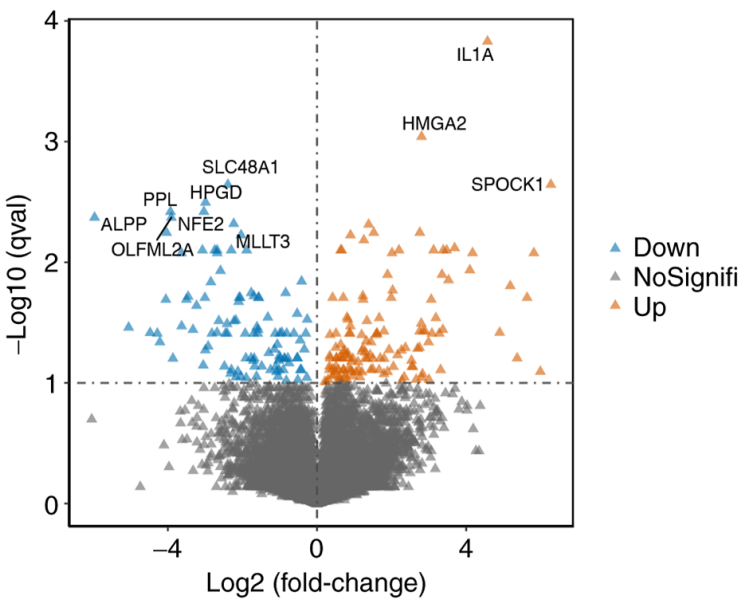

C

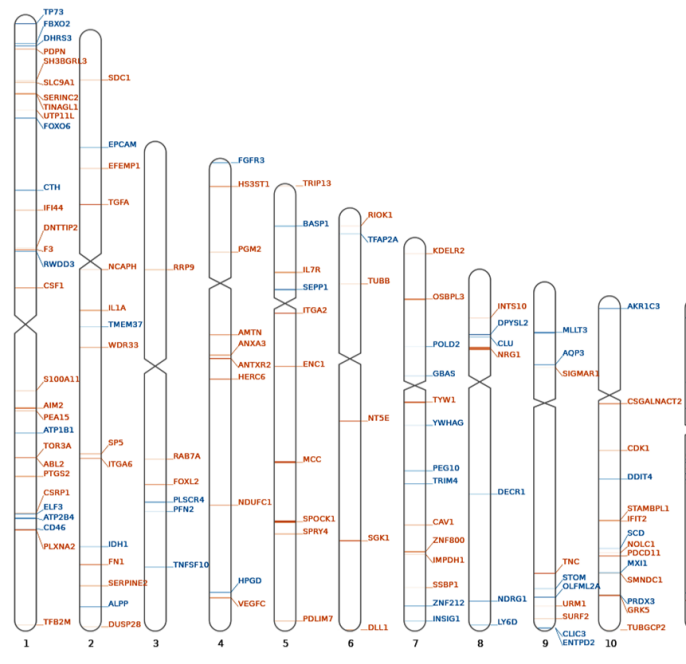

B

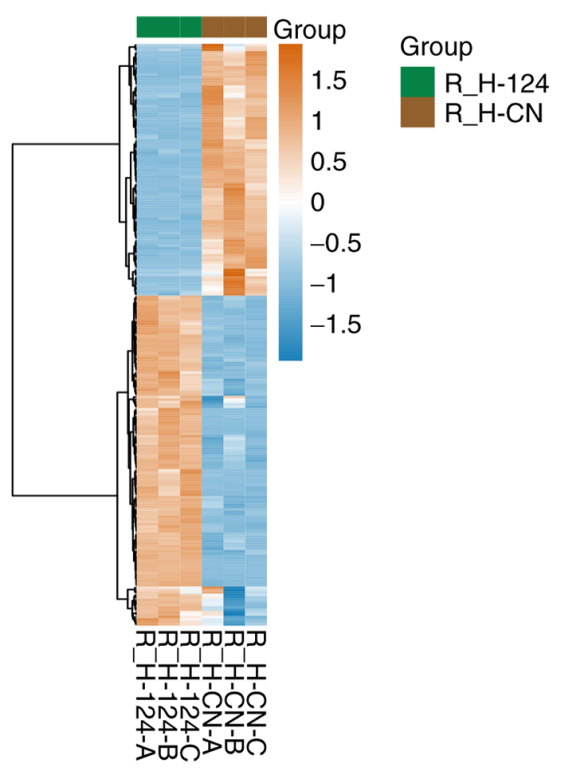

Figure 4. Analysis of differentially expressed genes (A) Volcano plot of the differentially expressed genes in human umbilical vein endothelial cells overexpressing miR-124 or a NC based on their expression fold change [thresholds, q val<0.1 and abs( $\log 2 \mathrm{FC})>0$ ]. (B) Heatmap and hierarchical clustering analysis of the differentially expressed genes. In samples that were not classified in advance, hierarchical cluster analysis revealed a different hierarchical clustering algorithm. (C) Distribution of differentially expressed genes associated with miR-124 overexpression on each chromosome. Chromosome distribution indicated that upregulated and downregulated mRNAs were spread on different chromosomal locations. Brown represents upregulated genes in microRNA 124-overexpression samples. Blue represents downregulated genes in microRNA 124-overexpression samples. R_H-124-A, R_H-124-B, R_H-124-C represent microRNA 124-overexpression samples; R_H-CN-A, R_H-CN-B, R_H-CN-C represent negative control samples R represents the use of R language for data analysis, $\mathrm{H}$ represents HUVEC cells, and A, B and C represent three different repeated samples. CN, negative control.

proliferation, migration and angiogenesis of HUVECs (49). In the present study, similar functions of miR-124 on PI3K/AKT pathways were identified in HUVECs. Therefore, in future studies, the role of the miR-124/PI3K/AKT axis in HUVEC angiogenesis warrants further study.

Although the present study provided evidence for the association between high miR-124 levels in peripheral blood and the occurrence of AMI, the potential mechanism in which miR-124 can regulate the PI3K/AKT signaling pathway and affect the proliferation of vascular endothelial cells remain unclear. Therefore, present results need to be interpreted with caution.

The current study constructed a connectivity map database between small molecules and pathways, and analyzed the topological properties of this map. Anisomycin and sanguinarine had the highest positive scores, meaning that Anisomycin and sanguinarine may suppress angiogenesis by inhibiting the PI3K/AKT signaling pathway, which was consistent with the results of previous reports indicating that they may be inhibitors of p38 MAPK activation (26,50). Additionally, rifabutin and quinostatin had the lowest scores, indicating that quinostatin is an inhibitor of the PI3K (51), while rifabutin is a wide spectrum antibiotic that is particularly effective on atypical and rifampicin-resistant mycobacteria (52). However, their potential roles in promoting angiogenesis and reducing the risk of myocardial infarction recurrence requires further study.

In addition, the present study has some limitations. A small cohort of patients was included, rendering the power of confidence insufficient. In future studies, the size of the cohort will need to be expanded. The present study also did not consider the blood pressure, a setting of diabetes, drug 


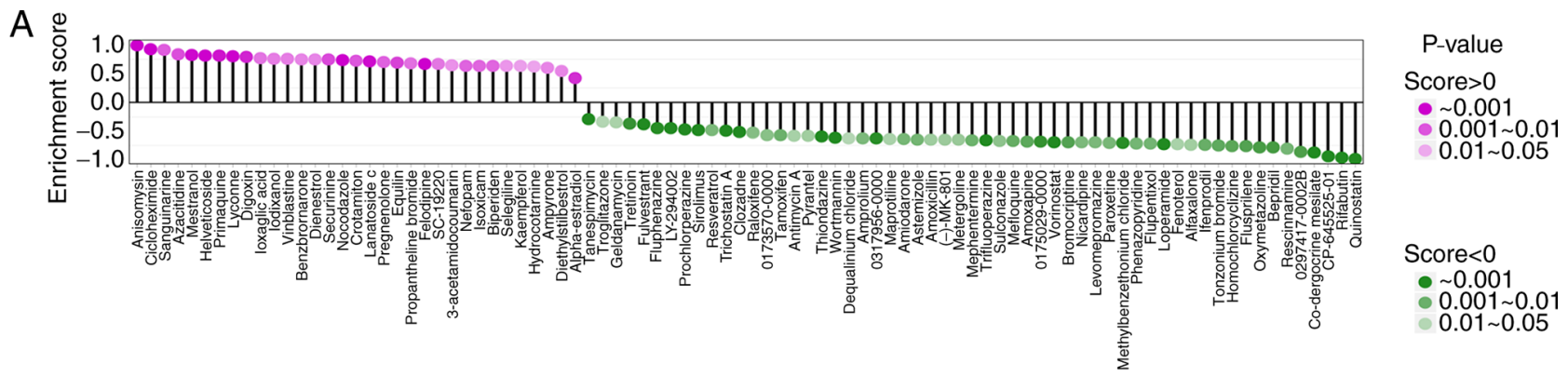

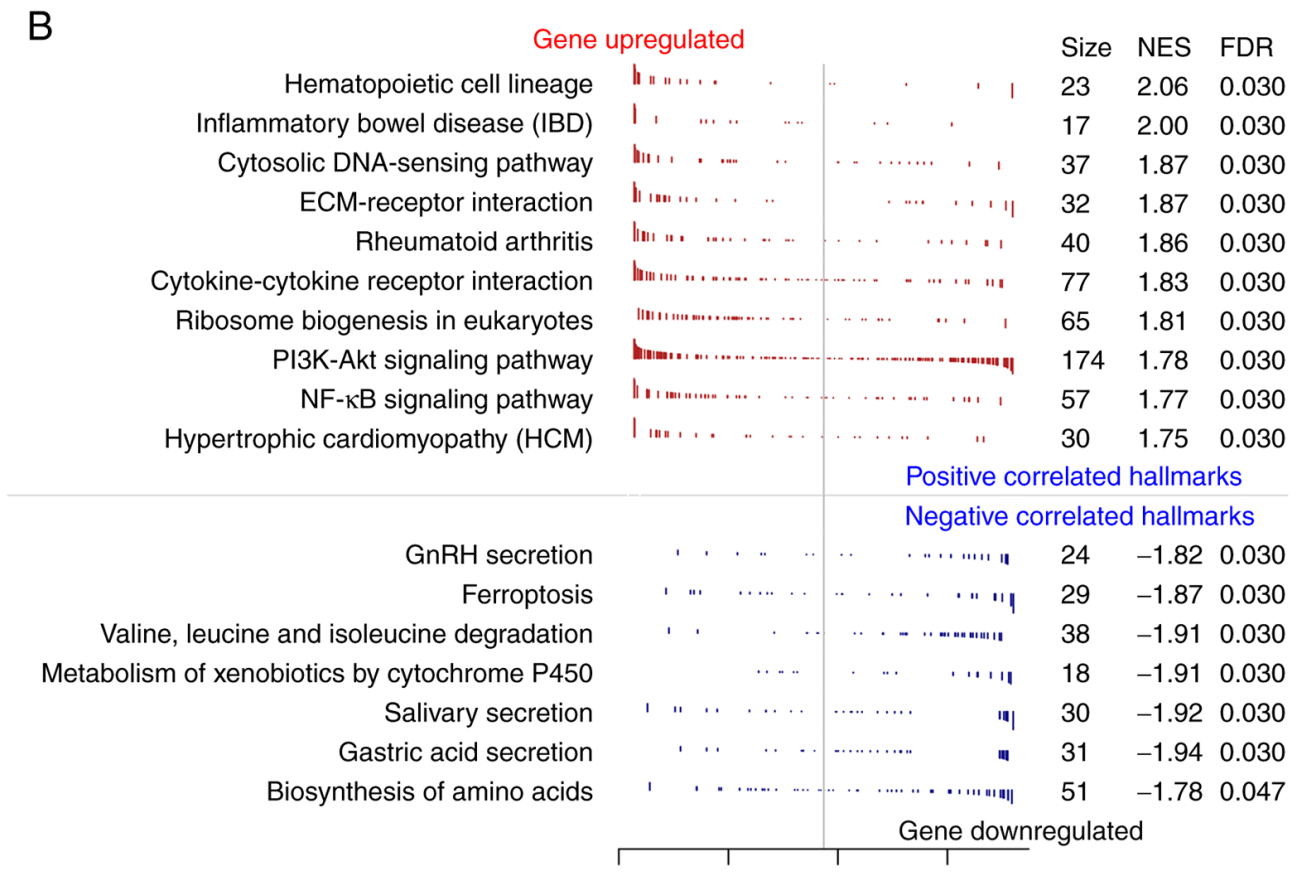

Figure 5. Drugs sensitivity analysis and GSEA enrichment. (A) Connectivity map analysis suggested that metformin generated a gene expression pattern concordant with HSF1 inactivation. Perturbagens inducing and reversing the biological state encoded in the query signature were assigned negative enrichment scores, respectively. The vertical line represents enrichment scores analysed by the cmap database (53). (B) Results of the GSEA reporting the most significant Kyoto Encyclopedia of Genes and Genomes pathways. (threshold values, NES >1, NES <-1 and FDR <0.05). GSEA, Gene Set Enrichment Analysis. NES, normalized enrichment score; FDR, false discovery rate.
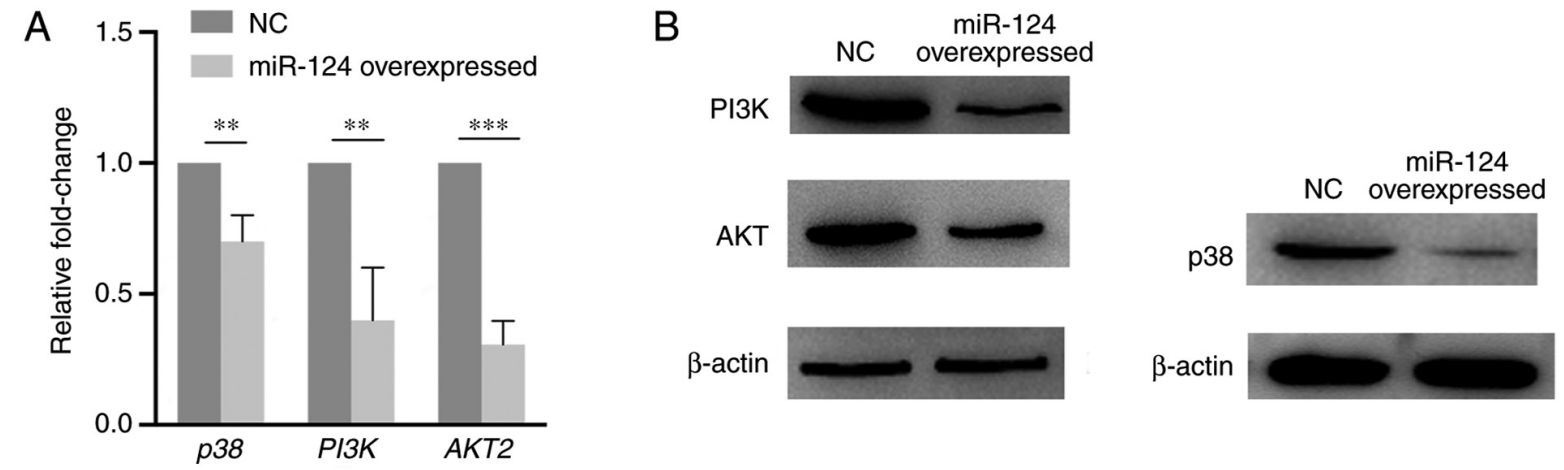

Figure 6. Effects of miR-124 overexpression on the expression of p38, PI3K and AKT in human umbilical vein endothelial cells. (A) The mRNA levels of p38, PI3K and AKT after miR-124 overexpression was measured by reverse transcription-quantitative PCR. ${ }^{* *} \mathrm{P}<0.01$ and ${ }^{* * *} \mathrm{P}<0.001$ (paired $\mathrm{t}$-test). (B) The protein expression levels of $\mathrm{p} 38$, PI3K and AKT after miR-124 overexpression was measured by western blotting. miR, microRNA; NC, negative control.

history or a history of other related diseases. Therefore, it is not possible to completely rule out that the observed differential expression of miR-124 in the blood samples was caused by the confounding factors aforementioned. Therefore, in future studies, stratified analysis will be conducted based on these potential confounding factors to determine the role of the miR-124/PI3K/AKT signaling pathway axis in angiogenesis to improve the recovery of patients with AMI. 


\section{Acknowledgements}

No applicable.

\section{Funding}

No funding was received.

\section{Availability of data and materials}

The datasets generated and/or analyzed during the current study are available in the Gene Expression Omnibus database repository (accession no. GSE180765), (https://www.ncbi.nlm. nih.gov/geo/query/acc.cgi?acc=GSE180765).

\section{Authors' contributions}

WM performed all the experiments. XZ performed clinic sample collection and preparation. YL performed bioinformatics analysis. All authors confirm the authenticity of all the raw data. All authors have read and approved the final manuscript.

\section{Ethics approval and consent to participate}

The study protocol was approved by the ethics committees of Weihai Central Hospital (approval no. WKY-2019-012). Informed consent was provided by all patients, and written permission was obtained before inclusion.

\section{Patient consent for publication}

Not applicable.

\section{Competing interests}

The authors declare that they have no competing interests.

\section{References}

1. Arora S, Stouffer GA, Kucharska-Newton AM, Qamar A, Vaduganathan M, Pandey A, Porterfield D, Blankstein R, Rosamond WD, Bhatt DL and Caughey MC: Twenty year trends and sex differences in young adults hospitalized with acute myocardial infarction. Circulation 139: 1047-1056, 2019.

2. Libby P: Mechanisms of acute coronary syndromes and their implications for therapy. N Engl J Med 368: 2004-2013, 2013.

3. Ferro G, Duilio C, Spinelli L, Liucci GA, Mazza F and Indolfi C: Relation between diastolic perfusion time and coronary artery stenosis during stress-induced myocardial ischemia. Circulation 92: 342-347, 1995.

4. Martín Giménez VM, de Las Heras N, Ferder L, Lahera V, Reiter RJ and Manucha W: Potential effects of melatonin and micronutrients on mitochondrial dysfunction during a cytokine storm typical of oxidative/inflammatory diseases. Diseases 9: 30, 2021.

5. Musher DM, Abers MS and Corrales-Medina VF: Acute infection and myocardial infarction. N Engl J Med 380: 171-176, 2019.

6. Ardekani AM and Naeini MM: The role of MicroRNAs in human diseases. Avicenna J Med Biotechnol 2: 161-179, 2010.

7. Condrat CE, Thompson DC, Barbu MG, Bugnar OL, Boboc A, Cretoiu D, Suciu N, Cretoiu SM and Voinea SC: MiRNAs as biomarkers in disease: Latest findings regarding their role in diagnosis and prognosis. Cells 9: 276, 2020.

8. Bartel DP: MicroRNAs: Target recognition and regulatory functions. Cell 136: 215-233, 2009.

9. Chen J and Wang DZ: MicroRNAs in cardiovascular development. J Mol Cell Cardiol 52: 949-957, 2012.
10. Silva DCPD, Carneiro FD, Almeida KC and Fernandes-Santos C: Role of miRNAs on the pathophysiology of cardiovascular diseases. Arq Bras Cardiol 111: 738-746, 2018.

11. Luo F, Wu P, Chen J, Guo Y, Wang J, Li X and Fang Z: ANGPTL3 possibly promotes cardiac angiogenesis through improving proangiogenic ability of endothelial progenitor cells after myocardial infarction. Lipids Health Dis 17: 184, 2018.

12. Su T, Shao X, Zhang X, Yang $C$ and Shao X: Value of circulating miRNA-1 detected within $3 \mathrm{~h}$ after the onset of acute chest pain in the diagnosis and prognosis of acute myocardial infarction. Int J Cardiol 307: 146-151, 2020.

13. Pinchi E, Frati P, Aromatario M, Cipolloni L, Fabbri M, La Russa R, Maiese A, Neri M, Santurro A, Scopetti M, et al: MiR-1, miR-499 and miR-208 are sensitive markers to diagnose sudden death due to early acute myocardial infarction. J Cell Mol Med 23: 6005-6016, 2019.

14. Yin Y, Lv L and Wang W: Expression of miRNA-214 in the sera of elderly patients with acute myocardial infarction and its effect on cardiomyocyte apoptosis. Exp Ther Med 17: 4657-4662, 2019.

15. Yücel EI and Sahin M: Fenretinide reduces angiogenesis by downregulating CDH5, FOXM1 and eNOS genes and suppressing microRNA-10b. Mol Biol Rep 47: 1649-1658, 2020.

16. Li X, Li Z, Zhu Y, Li Z, Yao L, Zhang L, Yuan L, Shang Y, Liu J and Li C: MiR-524-5p inhibits angiogenesis through targeting WNK1 in colon cancer cells. Am J Physiol Gastrointest Liver Physiol 318: G827-G839, 2020.

17. Lei Z, Klasson TD, Brandt MM, van de Hoek G, Logister I, Cheng C, Doevendans PA, Sluijter JPG and Giles RH: Control of angiogenesis via a VHL/miR-212/132 axis. Cells 9: 1017, 2020.

18. Livak KJ and Schmittgen TD: Analysis of relative gene expression data using real-time quantitative PCR and the 2(-Delta Delta C(T)) method. Methods 25: 402-410, 2001.

19. Blankenberg D, Gordon A, Von Kuster G, Coraor N, Taylor J and Nekrutenko A; Galaxy Team: Manipulation of FASTQ data with galaxy. Bioinformatics 26: 1783-1788, 2010.

20. Trapnell C, Roberts A, Goff L, Pertea G, Kim D, Kelley DR, Pimentel H, Salzberg SL, Rinn JL and Pachter L: Differential gene and transcript expression analysis of RNA-seq experiments with TopHat and cufflinks. Nat Protoc 7: 562-578, 2012.

21. Bray NL, Pimentel H, Melsted P and Pachter L: Near-optimal probabilistic RNA-seq quantification. Nat Biotechnol 34: 525-527, 2016.

22. Pimentel H, Bray NL, Puente S, Melsted P and Pachter L: Differential analysis of RNA-seq incorporating quantification uncertainty. Nat Methods 14: 687-690, 2017.

23. Love MI, Huber W and Anders S: Moderated estimation of fold change and dispersion for RNA-seq data with DESeq2. Genome Biol 15: 550, 2014.

24. Hao Z, Lv D, Ge Y, Shi J, Weijers D, Yu G and Chen J: RIdeogram: Drawing SVG graphics to visualize and map genome-wide data on the idiograms. PeerJ Comput Sci 6: e251, 2020.

25. Yu G, Wang LG, Han Y and He QY: ClusterProfiler: An R package for comparing biological themes among gene clusters. OMICS 16: 284-287, 2012.

26. Chai W, Wu Y, Li G, Cao W, Yang Z and Liu Z: Activation of p38 mitogen-activated protein kinase abolishes insulin-mediated myocardial protection against ischemia-reperfusion injury. Am J Physiol Endocrinol Metab 294: E183-E189, 2008.

27. Han F, Chen Q, Su J, Zheng A, Chen K, Sun S, Wu H, Jiang L, $\mathrm{Xu}$ X, Yang M, et al: MicroRNA-124 regulates cardiomyocyte apoptosis and myocardial infarction through targeting Dhcr24. J Mol Cell Cardiol 132: 178-188, 2019.

28. Guo ML, Guo LL and Weng YQ: Implication of peripheral blood miRNA-124 in predicting acute myocardial infarction. Eur Rev Med Pharmacol Sci 21: 1054-1059, 2017.

29. Lazar E, Benedek T, Korodi S, Rat N, Lo J and Benedek I: Stem cell-derived exosomes-an emerging tool for myocardial regeneration. World J Stem Cells 10: 106-115, 2018.

30. Sondergaard C, Hess DA, Maxwell DJ, Weinheimer C, Rosová I, Creer MH, Piwnica-Worms D, Kovacs A, Pedersen L and Nolta JA: Human cord blood progenitors with high aldehyde dehydrogenase activity improve vascular density in a model of acute myocardial infarction. J Transl Med 8: 24, 2010.

31. Makeyev EV, Zhang J, Carrasco MA and Maniatis T: The MicroRNA miR-124 promotes neuronal differentiation by triggering brain-specific alternative Pre-mRNA splicing. Mol Cell 27: 435-448, 2007.

32. Angelopoulou E, Paudel YN and Piperi C: MiR-124 and Parkinson's disease: A biomarker with therapeutic potential. Pharmacol Res 150: 104515, 2019. 
33. Sõber S, Laan M and Annilo T: MicroRNAs miR-124 and miR-135a are potential regulators of the mineralocorticoid receptor gene (NR3C2) expression. Biochem Biophys Res Commun 391: 727-732, 2010.

34. Hao Q, Zhang F, Wang Y, Li Y and Qi X: Cardiac contractility modulation attenuates chronic heart failure in a rabbit model via the PI3K/AKT pathway. Biomed Res Int 2020: 1625362 , 2020 .

35. Wang X, Chen L, Zhao X, Xiao L, Yi S, Kong Y, Jiang Y and Zhang J: A cathelicidin-related antimicrobial peptide suppresses cardiac hypertrophy induced by pressure overload by regulating IGFR1/PI3K/AKT and TLR9/AMPK $\alpha$. Cell Death Dis 11: 96, 2020.

36. Guo RX, Wei LH, Tu Z, Sun PM, Wang JL, Zhao D, Li XP and Tang JM: 17 beta-estradiol activates PI3K/Akt signaling pathway by estrogen receptor (ER)-dependent and ER-independent mechanisms in endometrial cancer cells. J Steroid Biochem Mol Biol 99: 9-18, 2006.

37. Chen J, Zacharek A, Cui X, Shehadah A, Jiang H, Roberts C, Lu M and Chopp M: Treatment of stroke with a synthetic liver $\mathrm{X}$ receptor agonist, TO901317, promotes synaptic plasticity and axonal regeneration in mice. J Cereb Blood Flow Metab 30: 102-109, 2010.

38. Zhou YH, Han QF, Gao L, Sun Y, Tang ZW, Wang M, Wang W and Yao HC: HMGB1 protects the heart against ischemiareperfusion injury via PI3K/AkT pathway-mediated upregulation of VEGF expression. Front Physiol 10: 1595, 2020.

39. Chen X, Wang R, Chen W, Lai L and Li Z: Decoy receptor-3 regulates inflammation and apoptosis via PI3K/AKT signaling pathway in coronary heart disease. Exp Ther Med 17: 2614-2622, 2019.

40. Cheng S, Zhang X, Feng Q, Chen J, Shen L, Yu P, Yang L, Chen D, Zhang H, Sun W and Chen X: Astragaloside IV exerts angiogenesis and cardioprotection after myocardial infarction via regulating PTEN/PI3K/Akt signaling pathway. Life Sci 227 82-93, 2019.

41. Xing X, Guo S, Zhang G, Liu Y, Bi S, Wang X and Lu Q: MiR-26a-5p protects against myocardial ischemia/reperfusion injury by regulating the PTEN/PI3K/AKT signaling pathway. Braz J Med Biol Res 53: e9106, 2020.

42. Coco D and Leanza S: A review on aorta mesenteric bypass in surgical management of mesenteric ischemia: Indications, techniques and outcomes. Maedica (Bucur) 15: 381-390, 2020.

43. van der Laan AM, Piek JJ and van Royen N: Targeting angiogenesis to restore the microcirculation after reperfused MI. Nat Rev Cardiol 6: 515-523, 2009.
44. Martini M, De Santis MC, Braccini L, Gulluni F and Hirsch E: PI3K/AKT signaling pathway and cancer: An updated review. Ann Med 46: 372-383, 2014.

45. Gao N, Zhang Z, Jiang BH and Shi X: Role of PI3K/AKT/mTOR signaling in the cell cycle progression of human prostate cancer. Biochem Biophys Res Commun 310: 1124-1132, 2003.

46. Zhu Y, Liu X, Zhao P, Zhao H, Gao W and Wang L: Celastrol suppresses glioma vasculogenic mimicry formation and angiogenesis by blocking the PI3K/Akt/mTOR signaling pathway. Front Pharmacol 11: 25, 2020.

47. Zhou DM, Sun LL, Zhu J, Chen B, Li XQ and Li WD: MiR-9 promotes angiogenesis of endothelial progenitor cell to facilitate thrombi recanalization via targeting TRPM7 through PI3K/Akt/ autophagy pathway. J Cell Mol Med 24: 4624-4632, 2020.

48. Peng H, Yang H, Xiang X and Li S: MicroRNA-221 participates in cerebral ischemic stroke by modulating endothelial cell function by regulating the PTEN/PI3K/AKT pathway. Exp Ther Med 19: 443-450, 2020.

49. Li Y, Xu Q, Shi M, Gan P, Huang Q, Wang A, Tan G, Fang Y and Liao H: Low-level laser therapy induces human umbilical vascular endothelial cell proliferation, migration and tube formation through activating the PI3K/Akt signaling pathway. Microvasc Res 129: 103959, 2020.

50. Niu X, Fan T, Li W, Xing W and Huang H: The anti-inflammatory effects of sanguinarine and its modulation of inflammatory mediators from peritoneal macrophages. Eur J Pharmacol 689: 262-269, 2012

51. Yang J, Shamji A, Matchacheep S and Schreiber SL: Identification of a small-molecule inhibitor of class Ia PI3Ks with cell-based screening. Chem Biol 14: 371-377, 2007.

52. Brughera M, Scampini G, Newman AJ, Castellino S, Sammartini U and Mazué G: Overview of toxicological data on rifabutin. Exp Toxicol Pathol 47: 1-9, 1995.

53. Tiedemann RE, Schmidt J, Keats JJ, Shi CX, Zhu YX, Palmer SE, Mao X, Schimmer AD and Stewart AK: Identification of a potent natural triterpenoid inhibitor of proteosome chymotrypsin-like activity and NF-kappaB with antimyeloma activity in vitro and in vivo. Blood 113: 4027-4037, 2009. International (CC BY-NC-ND 4.0) License. 